\title{
Guideline on fetal monitoring in labour
}

Fetal monitoring in labour could be done by:

- Intermittent auscultation (preferably by a hand held Doppler device)

- Intermittent or continuous electronic monitoring

Intermittent auscultation is recommended for low-risk women in spontaneous labour.

Electronic monitoring is recommended when:

- The baby is growth restricted

- There is significant meconium staining of amniotic fluid

- Abnormal fetal heart rate detected by intermittent auscultation

- Fresh vaginal bleeding

- Maternal pyrexia

- Use of oxytocin for augmentation or induction of labour

- Women with a scarred uterus

- Women on epidural analgesia

\section{Intermittent auscultation}

This could be done by using either a Pinnard's stethoscope or preferably a hand-held Doppler device.

Auscultation should be carried out immediately after a contraction for one full minute.

The maternal pulse should be palpated if there is suspected fetal bradycardia or any other FHR anomaly to differentiate the two heart rates.

The normal rate is between 110 - 160 beats per minute in a term fetus.

The frequency of auscultation should be as specified in the partogram.

\section{Electronic fetal monitoring (EFM)}

EFM is carried out by external cardiotocography (CTG).

The following are recommended at the commencement of a CTG.
1. The paper speed must be set at $1 \mathrm{~cm}$ per minute.

2. The date and time settings on the machine must be validated.

3. The CTG must be labeled with the mother's name, BHT number and date and time.

4. Maternal heart rate should be noted on the CTG.

5. The presence and the point at which the fetal heart rate is best heard must be delineated by auscultation and the probe placed at that point.

6. Ensure that the contraction probe is functioning properly and used for the recording.

7. The woman should be positioned in such a way that aortocaval compression is avoided.

8. It should be interpreted without delay and the categorization recorded as either normal or suspicious or pathological, as per Table 1, and signed by the responsible officer. The entry on the BHT must include a plan for management.

9. If the CTG is categorized as suspicious or abnormal, the Consultant must be informed.

10. For the management plan the overall clinical picture must be taken into account. e.g. the rate of progress of labour, presence or absence of fetal growth restriction, meconium staining of amniotic fluid and the evolution of the CTG abnormalities.

Table 1. Definitions of normal, suspicious and pathological FHR traces

Category Definition

Normal

An FHR trace in which all four features are classified as reassuring

Suspicious An FHR trace with one feature classified as non-reassuring and the remaining features classified as reassuring

Pathological An FHR trace with two or more features classified as non reassuring or one or more classified as abnormal 
Table 2. Classification of fetal heart rate patterns

\begin{tabular}{|c|c|c|c|c|}
\hline Feature & Baseline (bpm) & Variability (bpm) & Decelerations & Accelerations \\
\hline Reassuring & $110-160$ & $\geq 5$ & None & Present \\
\hline Non-reassuring & $\begin{array}{l}100-109 \\
161-180\end{array}$ & $\begin{array}{l}<5 \text { for } 40-90 \\
\text { minutes }\end{array}$ & $\begin{array}{l}\text { Typical variable } \\
\text { decelerations with } \\
\text { over } 50 \% \text { of contractions, } \\
\text { occurring for over } 90 \\
\text { minutes }\end{array}$ & $\begin{array}{l}\text { The absence of } \\
\text { accelerations with } \\
\text { otherwise normal } \\
\text { trace is of uncertain } \\
\text { significance }\end{array}$ \\
\hline Abnormal & $\begin{array}{l}<100 \\
>180 \\
\text { Sinusoidal } \\
\text { pattern } \geq 10 \\
\text { minutes }\end{array}$ & $<5$ for 90 minutes & $\begin{array}{l}\text { Either atypical variable } \\
\text { decelerations with over } \\
50 \% \text { of contractions or late } \\
\text { decelerations, both } \\
\text { for over } 30 \text { minutes }\end{array}$ & \\
\hline
\end{tabular}

\section{Further useful information on FHR patterns}

- If repeated accelerations are present with reduced variability, the FHR trace should be regarded as reassuring.

- True early uniform decelerations are rare and benign, and therefore they are not significant.

- Most decelerations that occur during labour are variable.

- If a bradycardia occurs in the baby for more than 3 minutes, urgent medical aid should be sought and preparations should be made to urgently expedite the birth of the baby, i.e. immediate commencement of cesarean section. This could include moving the woman to theatre if the fetal heart has not recovered by 9 minutes. If the fetal heart recovers within 9 minutes the decision to deliver should be reconsidered in conjunction with the woman if the post-recovery tracing is reassuring.

- A tachycardia in the baby of $160-180 \mathrm{bpm}$, where accelerations are present and no other adverse features appear, should not be regarded as suspicious. However, an increase in the baseline heart rate, even within the normal range, with other non-reassuring or abnormal features should increase concern. In such cases inquiry must be made to ascertain if the fetus was active during the recording.

When women are having continuous EFM, systematic assessment of above definitions and classification should be undertaken with every review.

During episodes of abnormal FHR patterns, if the woman is lying supine, advise her to adopt the left lateral position. 